\title{
A CHEMICAL STUDY OF WHEAT.
}

By G. B. Frankforter and E. P. Harding.

Rectived May J, 1899 .

WHEAT, from its great commercial importance, has been made the subject of a large number of chemical discussions. As a result of these investigations, much has been learned concerning its chemical composition, and several hitherto new substances have been described. The extreme difficulty, however, in obtaining these new compounds, together with their non-crystallinity, leaves some doubt as to the actual existence of some of them.

One of the objects which presented itself, in beginning this paper, was to verify, if possible, the existence of tritisecalin and cerealin ${ }^{1}$ and, if present, to isolate them. While the desire still exists, the numerous and perhaps less formidable questions which arose as the work progressed, led us somewhat from the original aim.

From an approximate analytical standpoint, wheat has been repeatedly examined. Konig ${ }^{2}$ has made an exhaustive examination of the European varieties, while Richardson ${ }^{3}$ has carefully examined those of otr own states and Canada. Throughout the whole of these analyses, only water, oil, carbohydrates, albuminoids and ash were estimated, together with the weight of the average wheat grain.

From over two hundred analyses, Richardson obtained the following averages:

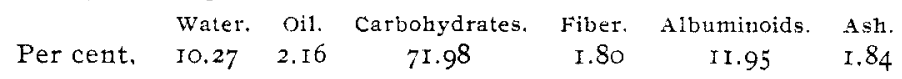

The average weight of 100 grains was 3.638 grams.

WHEAT OIL.

As above indicated, the quantity of oil in wheat has often been determined; but so far as we have been able to find, the oil itself has not been thoroughly studied. At the time of the beginning of this paper, the enormous quantities of wheat germ from the great mills of Minneapolis, had little or no value. It seemed important, therefore, that some commercial value should be found for this part of the wheat. As is well known, the germ

\footnotetext{
1. Compt. rend., 37, 775;38,505;42,1122;48,431

2 Nahrungs und Genussmittel.

3 Am. Chem. J., 6, 302 .
} 
of the wheat contains a very large per cent. of the oil, which was, therefore, obtained from that source.

Nowithstanding the fact that the oil has been determined in the germ and bran of the mills of Minneapolis, the improved methods of milling made it of interest to repeat some of these determinations. According to these analyses, ${ }^{\prime}$ the germ was divided into three distinct parts, varying materially in the amount of oil they contain. The following per cents. of oil were obtained :

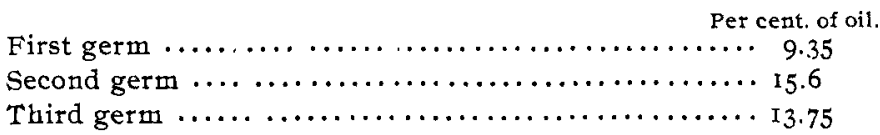

According to the best milling authorities here, no such distinction can be made at the present time. Several analyses of all the different samples which could be obtained, ran between eleven and twelve per cent. of oil. The average of all these analyses was II.6 per cent.

In the separation of the oil from the germ, several methods were tried. The simple extraction with ether was finally adopted as giving best results. The oil, when first extracted from the germ, has a golden-yellow color and a characteristic odor of freshly ground wheat. It has a specific gravity of 0.9292 at $15^{\circ} \mathrm{C}$, and 0.9374 at $0^{\circ} \mathrm{C}$. At $15^{\circ}$, it begins to change in appearance, becoming milky, and at $0^{\circ}$ it becomes a cloudy semisolid. Warmed at $100^{\circ} \mathrm{C}$., the above-mentioned odor is very pronounced and the oil changes color, becoming reddish brown. It is very soluble in ether, petroleum ether, chloroform, carbon disulphide, acetone and comparatively so in methyl and ethyl alcohols. It cannot be regarded as a drying oil, as it loses slightly in weight after exposure to the air for a month. After that time, the weight appears perfectly constant. The following table will show that the oil can not even be regarded as a semidrying oil. For the sake of comparison, duplicate drying experiments were made with pure linseed oil.

GaIN ( + ) AND Loss ( - ) IN PARTS PER HUNDRED AFTER

DRYING AT THE ORDINARY TEMPERATURE THE INDI-

CATED NUMBER OF DAYS.

\begin{tabular}{|c|c|c|c|c|c|c|c|}
\hline \multirow[b]{2}{*}{ Linseed oil } & \multicolumn{7}{|c|}{ Number of days. } \\
\hline & $\stackrel{5}{0.037}$ & $\begin{array}{c}10 \\
0.13\end{array}$ & $\begin{array}{l}15 \\
0.28\end{array}$ & $\begin{array}{c}20 \\
1.74\end{array}$ & $\begin{array}{r}25 \\
4.82\end{array}$ & $\begin{array}{c}30 \\
7.55\end{array}$ & $\begin{array}{c}35 \\
7.94\end{array}$ \\
\hline Wheat oil & 0.037 & 0.077 & O.I 5 & 0.24 & 0.30 & 0.37 & 0.37 \\
\hline
\end{tabular}




\begin{tabular}{lrrrrrrrr} 
& \multicolumn{1}{c}{40} & 45 & 50 & 55 & 60 & 65 & 70 & 75 \\
Linseed oil & 7.94 & 7.94 & 7.90 & 7.90 & 7.90 & 7.95 & 7.95 & 8.43 \\
Wheat oil & $0.4 \mathrm{I}$ & 0.42 & -0.22 & -0.26 & -0.28 & -0.28 & -0.30 & -0.42
\end{tabular}

The variation in the results between thirty-five and fifty days is undoubtedly due to outside physical conditions. Under exactly the same temperature, linseed oil will more than likely increase in weight uniformly, until the maximum of 8.5 per cent. is reached. Wheat oil, as indicated in the above table, can not even be regarded as a semidrying oil. Although a slight increase in weight was indicated during the first forty-five days, the loss by weight which followed, a little more than counterbalanced the gain. The oil, at the end of the above-stated time, appeared almost unchanged.

\section{VISCOSITY OF WHEAT OIL.}

The viscosity of wheat oil was taken at the various temperatures indicated below, as compared with pure rape oil. The following is a ratio between the two oils:

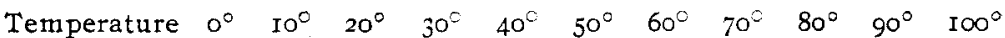
$\begin{array}{llllllllllll}\text { Wheat oil } & \text { I.43 } & \text { I.8I } & 2.57 & \text { I.92 } & \text { I.67 } & \text { I.48 } & \text { I.40 } & \text { I.39 } & \text { I.27 } & \text { I.20 } & \text { I.I4 }\end{array}$

While rape oil, which was taken as unity, was found to vary quite uniformly with increase of temperature, wheat oil increased more rapidly from $0^{\circ}$ to $20^{\circ}$ than from $20^{\circ}$ to $100^{\circ} \mathrm{C}$., making the maximum ratio between the two oils at $20^{\circ} \mathrm{C}$. This ratio may be represented by the following curve in which the temperature is the abcissa, and the relative viscosity, the ordinate :

2

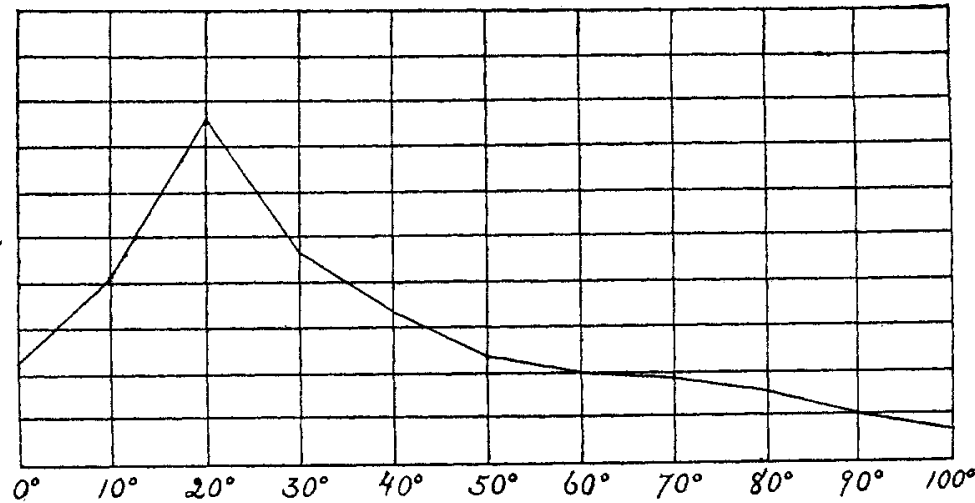

Fig. - -viscosity Curve of Wheat Oil, Rape Oil as Unity. 
INDEX OF REFRACTION.

The index of refraction of wheat oil was taken at different temperatures by the Pulfrich refractometer. The following is a comparison of wheat oil with corn and linseed oils :

\begin{tabular}{|c|c|c|c|c|c|c|c|}
\hline Temperature & $20^{\circ}$ & $30^{\circ}$ & $40^{\circ}$ & $50^{\circ}$ & $60^{\circ}$ & $70^{\circ}$ & $80^{\circ}$ \\
\hline $\begin{array}{l}\text { Corrected index } \\
\text { of wheat oil }\end{array}$ & & & 1.47181 & I. 46858 & .46450 & I. $46 \mathrm{I} 6 \mathrm{I}$ & I.4586 \\
\hline Corn oil & I. 47789 & I. 47436 & r. 47053 & 1.46728 & 1.46360 & I. 46002 & I. 45642 \\
\hline Linseed oil & I. $4855^{8}$ & I. 48072 & I. $4773^{\circ}$ & 1.47329 & 1.46954 & I. 46697 & I. $4628 \mathrm{I}$ \\
\hline
\end{tabular}

The above readings were taken with wheat oil, just as it was obtained from the germ, by extracting with ether. The oil, therefore, contained all the substances occurring in the germ, which are soluble in ether. Some of these, it was found, could be removed by redissolving in ether and evaporating rapidly, while nearly all of these substances, including the paracholesterol, were removed by treating with alcohol. The oil thus purified had changed its properties slightly. The refractive index had changed somewhat, giving at a temperature of $20^{\circ}$, $30^{\circ}$, and $40^{\circ} \mathrm{C}$., corrected indices of I.48325, I.47936, and I.47447 respectively.

THE ABSORPTION SPECTRUM OF WHEAT OIL.

So far as we can learn, very little has been done on the absorption spectra of the oils. Several attempts have been made to classify the oils with regard to their absorption bands, but as these bands are in many cases due to impurities in the oil, the classification is a rather imperfect one. According to Vogel, who briefly studied a few of the organic substances, these bands are nearly always due to the presence of foreign matter, as chlorophyl or other coloring substance, for by removing these coloring substances, the absorption bands disappear. This statement seems to be true within certain limits, in the case of the oils. A few of the oils undoubtedly owe their spectra to chlorophyl or some other coloring-matter. Many of them, however, have spectra entirely independent of foreign substances. Doumer, ${ }^{2}$ in a spectral examination of some of the oils, divided them into four distinct classes which were represented by the following characteristics:

1 Vogel's spectralanalyse, Vo1. I.

2 Analyst (1885), 148. 
Class I, oils showing bands due to chlorophyl.

Class II, oils without absorption bands.

Class III, oils absorbing the chemical rays of the spectrum, the red, orange, yellow and a part of the green remaining unchanged.

Class IV, oils showing absorption bands in different parts of the scale.

According to this classification, linseed, mustard and rape oils belong to Class III. A glance at the following spectra will indicate that linseed together with wheat and corn oils belong to class IV. We have found that the spectrum depends largely upon the length of the tube used. Thus with proper length tubes these three common oils may belong to Class III, while with different tubes they nay be regarded as members of Class IV. It seems evident, if this classification is to be used, that the length of the tube must be taken into consideration. Thus, wheat oil, with a $200 \mathrm{~mm}$. tube, gives a rather indistinct spectrum, while with a $100 \mathrm{~mm}$. tube, the spectrum is characterized by two very distinct bands, as seen below. Corn and linseed oils, with the same length tube, appear to belong to Class III. However, with a tube double the length, they undoubtedly belong to Class IV as will be seen by the following charts:

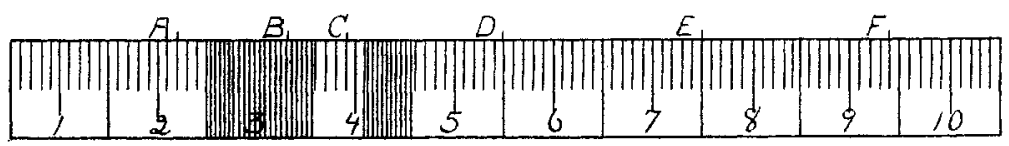

Fig. 2,-Wheat Oil.

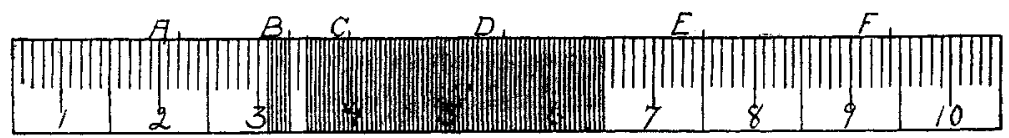

Fig. 3.-Corn Oil.

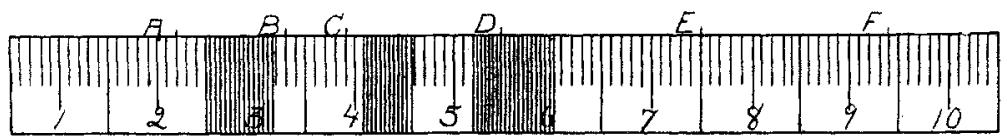

Fig. 4-Linseed Cil. 


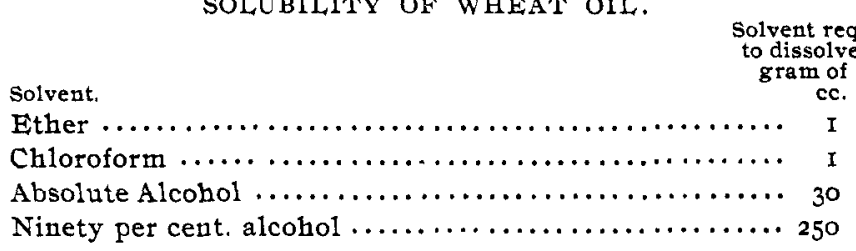

Some difficulties were at first experienced in determining the solubility, owing to the fact that a small quantity of unsaponifiable substance was soluble with difficulty in absolute, and almost insoluble in ninety per cent., alcohol. The oil was at first purified by dissolving in a small quantity of absolute alcohol and filtering. The tests were made on the oil thus purified.

\section{SAPONIFICATION VALUE OF WHEAT OIL.}

The saponification value of wheat oil was determined according to Köttstorfer's method which represents the number of milligrams of potassium hydroxide required to saponify one gram of the oil. The results obtained agree best with the values obtained from the semidrying oils, notwithstanding the fact that the drying experiments would not permit it to be classed in that group.

The following is the result of four determinations as compared with the results obtained from typical semidrying oils:

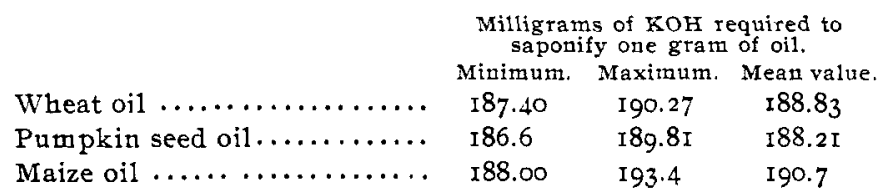

In these determinations, freshly extracted oil was used, inasmuch as oil extracted for some time gave varying results.

THE IODINE VAIUE.

The iodine value of wheat oil was determined according to Hübl's method. Three determinations gave the following results :

I. 0.1998 gram of the oil absorbed 232 milligrams of iodine. II. 0.1866 gram of the oil absorbed 216 milligrams of iodine. III. 0.1505 gram of the oil absorbed 74 milligrams of iodine. 


\begin{tabular}{cccc}
\multicolumn{4}{c}{ Per cent. of iodine found. } \\
I. II. & II. & III. & Average. \\
II6.16 & II 5.75 & II 5.03 & II5.64
\end{tabular}

Pumpkin seed gave a mean of $\ldots \ldots \ldots \ldots \ldots \ldots \ldots \ldots$ I2I.

Maize oil gave a mean of $\ldots \ldots \ldots \ldots \ldots \ldots \ldots \ldots+122.27^{1}$

THE ACID VALUE.

The acid value of wheat oil was determined by the common method of titrating a known quantity of the oil with decinormal alcoholic potassium hydroxide. Three determinations gare the following results :

I. I.594 grams of the oil required 0.066 gram of potassium hydroxide.

II. 1.56 I6 grams of the oil required 0.06284 gram of potassium hydroxide.

III. I.268 grams of the oil required 0.05145 gram of potassium hydroxide.

Acid value $\ldots \ldots \ldots \ldots \ldots \ldots \ldots \ldots+4$ I. $\quad \begin{array}{cc}\text { II. } & \text { III. } \\ 40.2 & 40.5\end{array}$

DETERMINATION OF GLYCEROL.

Glycerol was determined by the oxidation process, that is, the oxidation of glycerol to oxalic acids by means of alkaline potassium permanganate according to the following reaction:

$$
\mathrm{C}_{3} \mathrm{H}_{8} \mathrm{O}_{3}+3 \mathrm{O}_{2}=\mathrm{C}_{2} \mathrm{H}_{2} \mathrm{O}_{4}+\mathrm{CO}_{2}+3 \mathrm{H}_{2} \mathrm{O} \text {, }
$$

and the estimation of oxalic acid, from which glycerol can be determined. The method as worked out by Benedict and Zsigmondy was used, and is briefly as follows: Two or three grams of the oil were saponified with potassium hydroxide in methyl alcohol. The alcohol was removed by evaporation, the soap dissolved in hot water and decomposed by hydrochloric acid. The whole mass was then warmed until the fatty acids had collected on the surface. These acids were filtered off and the filtrate carefully neutralized with potassium hydroxide. Ten grams of the hydroxide were now added and an excess of permanganate, or until the liquid appeared blue or dark red. The excess of permanganate was destroyed by sulphurous acid and the whole solution again filtered. The filtrate obtained was acidified with acetic acid, diluted to a liter, heated to the boilingpoint, and the oxalic acid precipitated by calcium chloride.

This Journal, 20, 948 . 
From the calcium oxalate formed, the amount of oxalic acid and hence the per cent. of glycerol can be calculated. Five analyses gave the following results :

I. 2.7696 grams of oil gave 0.2024 gram of glycerol.

II. 2.9074 grams of oil gave 0.2145 gram of glycerol.

III. 2.8778 grams of oil gave 0.2124 gram of glycerol.

IV. 2. I640 grams of oil gave 0.1557 gram of glycerol.

V. 3.4940 grams of oil gave 0.263 I gram of glycerol.

$$
\begin{array}{rlllcc} 
& \text { I. } & \text { II. } & \text { III. } & \text { IV. } & \text { v. } \\
\text { Per cent. glycerol...... } & 7.34 & 7.29 & 7.38 & 7.2 & 7.53 \\
\text { DETERMINATION } & \text { OF LECITHIN } & \text { IN WHEAT OIL. }
\end{array}
$$

Frequent references have been made to the occurrence of lecithin in wheat, but no quantitative determinations have been made, so far as we can learn. As the base was apparently abundant in the oil, determinations were made, using the common phosphoric acid method. A known quantity of the oil was saponified with a concentrated solution of potassium hydroxide and heated on a water-bath until all the alcohol had been removed. The soap thus formed was decomposed with hydrochloric acid and the fatty acids filtered off. The filtrate, which contained all the phosphorus, was evaporated to dryness and fused with potassium hydroxide and potassium nitrate. From the fused mass the phosphoric acid was determined and the lecithin calculated. Two determinations gave the following results :

I. $23.185^{8}$ grams of oil gave 0.0697 gram magnesium pyrophosphate.

II. $27.45 \mathrm{I} 4$ grams of oil gave 0.079 gram magnesium pyrophosphate.

Calculated for lecithin $\ldots \ldots \ldots \ldots 2.03 \quad$ I. $\quad$ II.

In making the above calculations, we have assumed that the lecithin was an oleyl rather than a stearyl ester. This assumption was made from the fact that in the analysis, little or no stearic acid was found in the oil. The formula used therefore, was

$$
\mathrm{C}_{42} \mathrm{H}_{84} \mathrm{O}_{9} \mathrm{NP} \text {. }
$$


PARACHOLESTEROL IN WHEAT OII.

Cholesterol was first obtained from wheat by Ritthausen. ${ }^{1}$ By extracting the gluten, as well as the fats in wheat with ether, he obtained, on evaporating off the ether, a crystalline substance which had very similar properties to the cholesterol of Beneke."

It was afterwards regarded as identical with an isomeric form of cholesterol discovered by Hesse, ${ }^{3}$ and called phytosterol.

In the beginning of our work on this substance, we obtained a meiting-point which compared so well with phytosterol, that, with Koenig, ${ }^{4}$ we regarded the two substances as identical.

However, after carefully recrystallizing several times, the melting-point had risen enough to make it doubtful if the substance in wheat were phytosterol. The melting-point of phytosterol, as given by Hesse and others, is $132^{\circ}-133^{\circ} \mathrm{C}$., while the purified substance from wheat, melted at $134^{\circ}-135^{\circ} \mathrm{C}$. A much greater difference, however, was observed in a comparison of the wheat cholesterol with the common or animal cholesterol, the melting-point of which ranges from $140^{\circ}$ to $148^{\circ} \mathrm{C}$. These two substances, to neither of which wheat cholesterol belongs, were all which were known to the earlier authorities, and according to Maly, 'these two substances were identical. However, later investigations seem to prove beyond a doubt, that several different forms of cholesterol exist. Reinke and Rodewald $^{6}$ found in protoplasm of aethalium septicum, a substance to which, after examination, they gave the name paracholesterol. Later, Schulze and Barbieri ${ }^{7}$ repeated much of the work which had previously been done, and showed that no less than five different isomeric forms of cholesterol existed, or two more than had heretofore been described. These isomeric forms, together with their melting-points and optical activities, are given in the following table:

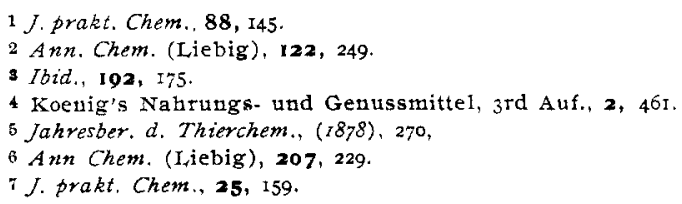


ISOMERIC FORMS OF CHOLESTEROL.

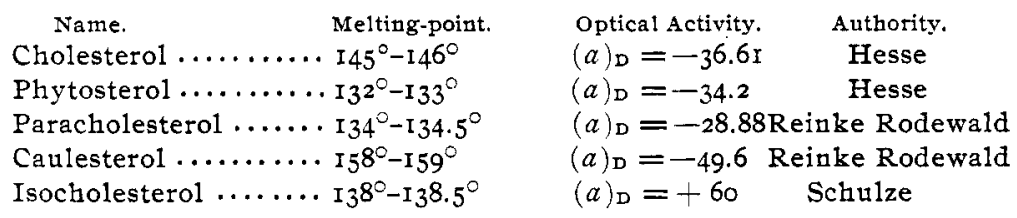

As will be seen from the above table, four of these forms of cholesterol are laevo-, while the fifth is dextrorotatory, thus affording a means of distinguishing between isocholesterol and the other members. In fact, the differences between the other members, taking into consideration the melting-points, are sufficient to readily distinguish between them. A glance at this table will enable us to identify the cholesterol in wheat as paracholesterol. The melting-points are exactly the same while the planes of rotation are almost the same, as will be seen by the comparison given below :

2.7 grams of paracholesterol gave $(a)_{1} 28.88$, melting-point I $34.5^{\circ}$.

1.7948 grams of paracholesterol gave $(a)_{D} 27.24$, melting-point I $34.5^{\circ}$.

I. 3848 grams of wheat cholesterol gave $(a)_{D} 26.9$, melting-point I $34.5^{\circ}$.

Taking into consideration the variation in the plane of polarization obtained by Reinke and Rodewald with different amounts of paracholesterol, the results obtained with wheat cholesterol will check exactly with paracholesterol.

\section{DETERMINATION OF PARACHOLESTEROL.}

The methods given for the extraction of these isomeric forms of cholesterol are very much the same. The method in common use is as follows: The substance containing the cholesterol is first saponified with potassium hydroxide and the cholesterol extracted by ether. The ether usually dissolves some other substances besides the cholesterol. These impurities are removed by again treating with potassium hydroxide, again extracting with ether and finally recrystallizing from alcohol. Bömer ${ }^{1}$ avoided this tedious process of evaporation, by extracting with

1 Ztschr. Unters. d. Nahyungs-und Genussmittel, (I898), 3 I. 
ether from aqueous solution. This method was found objectionable, on account of the large quantity of ether necessary to obtain perfect extraction.

Raumer' used good clean beach sand after saponification, the object being to assist in both evaporation and extraction.

This method will give good results, provided the sand is clean. Juckenack and Hilger ${ }^{2}$ suggest the use of sodium lydroxide instead of potassium hydroxide, and filter-paper instead of sand.

The above methods all give good results, the principal difference being in the time and material required. The method which we adopted, was that involving the saponification with alcoholic potassium hydroxide and extracting the dried soap with ether in a Soxhlet extractor. Five analyses gave the following results :

I. 20.0000 grams of oil gave 0.5216 gram of paracholesterol. II. $9.33^{80}$ grams of oil gave 0.2209 gram of paracholesterol. III. 10.3332 grams of oil gave 0.25 IO gram of paracholesterol. IV. IO.I3I4 grams of oil gave 0.25 IO gram of paracholesterol. V. I IO.3000 grams of oil gave 2.7573 grams of paracholesterol.

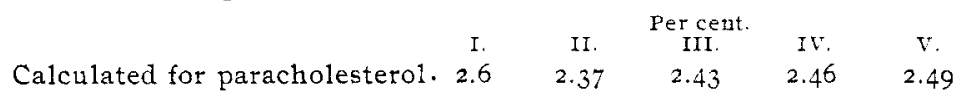

THE FLAIDIN METHOD.

Among the new methods tried, was one which involved the elaidin reaction, and which, carefully carried out, gave fairly good results. The method of operating was as follows: About twenty grams of the oil were transferred to a tall beaker, ten grams of mercury added, then ten cc. of strong nitric acid, drop by drop. The temperature was kept between $8^{\circ}$ and $10^{\circ}$, on account of the violent reaction between the mercury and the nitric acid at a higher temperature. When the mercury had quite dissolved, the beaker was gently warmed, in order to remove the excess of oxides of nitrogen. The whole mass was then treated with an excess of alcoholic potassium hydroxide, evaporated to dryness on a water-bath, and extracted with ether On evaporating off the ether, paracholesterol was obtained in comparatively pure form. By recrystallizing from alcohol, a very pure form was obtained.

1 Ztschr. angew. Chem., (1808). 555.

2 Arch. Pharm., 236, 367. 
Two determinations gave the following results:

I. 25.64 grams of oil gave 0.464 gram of paracholesterol.

II. 15.792 grams of oil gave 0.3474 gram of paracholesterol.

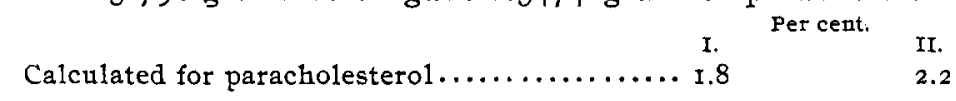

Although the paracholesterol was obtained in a comparatively pure form by this method, considerable difficulty was experienced in evaporating to dryness, several hours being required. It was also observed, that when more than the above-mentioned anount of nitric acid was used, the quantity of paracholesterol was diminished. Thus with double the quantities of nitric acid and mercury only four-tenths per cent., and with three times the quantity of acid and mercury only a trace, of the paracholesterol was obtained. Just why this diminution in the quantity of paracholesterol should occur, is not definitely known. The probabilities are, however, that it forms, with the excess of nitric acid, a nitrolic acid according to the common reaction.

\section{THE ESTIMATION OF ARSENIC IN PARIS GREEN.}

BY THORN SMITH.

Received May 6, 1899 .

I selecting a method for the analysis of the common insecti1 cide, Paris green, some difficulty was encountered in finding one suitable to the ordinary requirements of rapidity and corresponding accuracy. In fact the only methods of which record was found are as follows:

First, by direct precipitation of the arsenic as magnesium pyroarsenite by means of magnesia mixture or what would be embodied in the same method, a first separation of the sulphides of arsenic and copper, by means of hydrogen sulphide, and a consequent tedious separation of the arsenic from the copper by means of ammonium sulphide, followed by the precipitation of the arsenic as at first. This latter modification is given in the majority of text-books, which mention the subject at all. Its objection is the large amount of time consumed in the operation and the danger of loss in separating the arsenic from the copper.

The second method known as the "Pearce" or "McCay" method is perhaps just as difficult with the added objection that such small amounts of the material are permitted in the pro- 